\title{
MAGILL-TYPE THEOREMS FOR MAPPINGS
}

\section{GIORGIO NORDO and BORIS A. PASYNKOV}

\author{
Received 9 December 2002
}

\begin{abstract}
Magill's and Rayburn's theorems on the homeomorphism of Stone-Čech remainders and some of their generalizations to the remainders of arbitrary Hausdorff compactifications of Tychonoff spaces are extended to some class of mappings.
\end{abstract}

2000 Mathematics Subject Classification: 54C05, 54C20, 54D40, 54D50.

1. Introduction. In 1968, Magill [8] proved the following theorem.

THEOREM 1.1. For two locally compact Tychonoff spaces $X$ and $Y$, the Stone-Čech remainders (i.e., the remainders of the Stone-Čech compactifications) $\beta X \backslash X$ and $\beta Y \backslash Y$ are homeomorphic if and only if the posets $K(X)$ and $K(Y)$ of all Hausdorff compactifications of $X$ and $Y$ are isomorphic.

In 1973, Rayburn [12] gave the following definition.

DEFINITION 1.2. A Tychonoff space $X$ is called $k$-absolute if $\beta X \backslash X$ is a $k$-space.

It is proved in [12] that a Tychonoff space $X$ is $k$-absolute if and only if $c X \backslash X$ is a $k$-space for some $c X \in K(X)$ and if and only if $c X \backslash X$ is a $k$-space for any $c X \in K(X)$.

All locally compact Tychonoff spaces are $k$-absolute because their Stone-Čech remainders are compact. Consequently, the following Rayburn's theorem generalizes one half of Theorem 1.1.

THEOREM 1.3. For any pair of $k$-absolute spaces $X, Y$, if the posets $K(X)$ and $K(Y)$ are isomorphic, then the Stone-Čech remainders $\beta X \backslash X$ and $\beta Y \backslash Y$ are homeomorphic.

We note that the second half of Theorem 1.1 cannot be generalized to $k$-absolute spaces (see [12, Example (B)]).

In [2], both Theorems 1.1 and 1.3 are generalized to arbitrary compactifications of two Tychonoff spaces.

This paper is devoted to an extension of Theorems 1.1 and 1.3 and their generalizations (given in [2]) to the class of WZ-mappings (in particular, closed mappings) from a locally compact Tychonoff space or a $k$-absolute space to a compact Hausdorff space. Although, even for this rather narrow class of mappings, the formulations of corresponding theorems look rather complicated, the examples presented in Section 4 show that more simple approaches are not sufficient. 
We note that results concerning the extension of Theorem 1.1 to mappings are also contained in [5], but they are different from ours (see the remark at the end of this paper).

2. Preliminaries. Throughout this paper, space will mean a topological space and mapping will mean a continuous function. Terms and undefined concepts are used as in [4]. In this section, we recall some definitions and results from [2]. Some additional notions concerning fibrewise general topology (FGT) can be found in [9, 10].

DeFinition 2.1. Let $X, Y, Z$ be spaces and $\lambda: X \rightarrow Y, \mu: X \rightarrow Z$ mappings. We say that $\lambda$ is equivalent to $\mu$ and we will write $\lambda \equiv \mu$ if there exists some homeomorphism $h: Y \rightarrow Z$ such that $\mu=h \circ \lambda$.

Evidently, the homeomorphism $h$ is unique.

We will identify equivalent mappings, and so we can consider the set $\mathscr{C}(X)$ of all the continuous maps from a fixed space $X$ onto other spaces.

Definition 2.2. Let $\lambda, \mu \in \mathscr{b}(X)$. We say that $\lambda$ follows $\mu$ and we will write $\lambda \geq \mu$ if there exists some continuous mapping $h: Y \rightarrow Z$ such that $\mu=h \circ \lambda$.

It is evident that $(\mathscr{C}(X), \geq)$ is a poset.

DEFINITION 2.3. Let $\lambda \in \mathscr{C}(X)$. We say that

(i) $\lambda$ is simple if there exists a unique point $t_{\lambda} \in \lambda(X)$ such that $\left|\lambda^{-1}\left(\left\{t_{\lambda}\right\}\right)\right|>1$ and $\left|\lambda^{-1}(\{t\})\right|=1$ for every $t \in \lambda(X) \backslash\left\{t_{\lambda}\right\}$;

(ii) $\lambda$ is finite simple if there exists a nonempty finite set $T \subset \lambda(X)$ of points such that $\left|\lambda^{-1}(\{t\})\right|>1$ for every $t \in T$ and $\left|\lambda^{-1}(\{t\})\right|=1$ for every $t \in \lambda(X) \backslash T$.

We will suppose from this moment that $X$ is a Hausdorff space and that $\mathscr{P}(X)$ denotes the poset (as a subposet of $\mathscr{C}(X)$ ) of all perfect onto mappings of $X$. Clearly, $\lambda(X)$ is Hausdorff for any $\lambda \in \mathscr{P}(X)$.

Definition 2.4. A mapping $\lambda \in \mathscr{P}(X)$ is called a dual point if it is simple and $\left|\lambda^{-1}\left(\left\{t_{\lambda}\right\}\right)\right|=2$.

Let $\mathscr{D}=\mathscr{D}(X)$ denote the set of all dual points of $\mathscr{P}(X)$ and $\mathscr{F} \mathscr{Y}(X)=\{\lambda \in \mathscr{P}(X)$ : $\lambda$ is finite simple $\} \cup\left\{\operatorname{id}_{X}\right\}$.

DEFINITION 2.5. A family $\mathscr{F} \subset \mathscr{D}$ is said to be a 3-vertex family if for any distinct $\alpha, \beta \in \mathscr{F}$ there exists some $\gamma \in \mathscr{D} \backslash \mathscr{F}$ such that $\gamma>\inf \{\alpha, \beta\}$.

DEFINITION 2.6. A 3 -vertex family $\mathscr{F} \subset \mathscr{D}$ is called a point family if it is maximal (i.e., if there is no 3 -vertex family properly containing $\mathscr{F}$ ).

LEMMA 2.7. If F is a 3-vertex family consisting of more than one element, then the set $X_{\mathscr{F}}=\bigcap\left\{\lambda^{-1}\left(\left\{t_{\lambda}\right\}\right): \lambda \in \mathscr{F}\right\}$ is a single point (which will be denoted by $J_{\mathscr{F}}\left(\mathscr{F}_{F}\right)$ ).

DEFINITION 2.8. Let $\mathscr{I} \subset \mathscr{P}(X)$ such that $\mathscr{F} \mathscr{Y}(X) \subset \mathscr{I}$ and $x \in X$. We put $K_{\mathscr{I}}(x)=\{\delta \in$ $\left.\mathscr{D}: x \in \delta^{-1}\left(\left\{t_{\delta}\right\}\right)\right\}$.

LEMMA 2.9. If $|X|>2$, then $K_{\mathscr{I}}(x)$ is a point family. 
Evidently, for $|X|>2$,

$$
\begin{gathered}
J_{\mathscr{I}}\left(K_{\mathscr{I}}(x)\right)=x \text { for any } x \in X, \\
K_{\mathscr{I}}\left(J_{\mathscr{I}}(\mathscr{F})\right)=\mathscr{F} \quad \text { for any point family } \mathscr{F}_{\mathscr{F}} \subset \mathscr{D} .
\end{gathered}
$$

In [2], dual points are characterized only by means of the order in $\mathscr{I}$. It follows from this that if, for Hausdorff spaces $X_{j}$, we have $\mathscr{F} \mathscr{Y}\left(X_{j}\right) \subset \mathscr{I}_{j} \subset \mathscr{P}\left(X_{j}\right)$ with $j=1,2$ and $i: \mathscr{I}_{1} \rightarrow \mathscr{I}_{2}$ is a poset isomorphism, then $i\left(K_{\mathscr{I}_{1}}(x)\right)$ is a point family in $\mathscr{I}_{2}$.

We recall [2] that a one-to-one mapping $f: X \rightarrow Y$ between Hausdorff spaces is called a $k$-homeomorphism if $f$ is continuous on compact subsets of $X$ and its inverse $f^{-1}$ is continuous on compact subsets of $Y$. Clearly, a $k$-homeomorphism between $k$-spaces is a homeomorphism.

THEOREM 2.10. Let $X_{j}$ be a Hausdorff space and $\mathscr{P}\left(X_{j}\right)$ the set of all perfect onto mappings of $X_{j}$ (with $\left.j=1,2\right)$. If $X_{1}$ and $X_{2}$ are $k$-homeomorphic and they are $k$-spaces, then $\mathscr{P}\left(X_{1}\right)$ and $\mathscr{P}\left(X_{2}\right)$ (and so $\mathscr{F} \mathscr{S}\left(X_{1}\right)$ and $\mathscr{F} \mathscr{S}\left(X_{2}\right)$ ) are isomorphic. Let $\mathscr{F} \mathscr{Y}\left(X_{j}\right) \subset \mathscr{I}_{j} \subset \mathscr{P}\left(X_{j}\right)$ for $j=1,2$. If $\Phi_{1}$ and $\Phi_{2}$ are poset isomorphic, then $X_{1}$ and $X_{2}$ are $k$-homeomorphic, and if, additionally, $X_{1}$ and $X_{2}$ are $k$-spaces, then they are homeomorphic. More precisely, if $i: I_{1} \rightarrow \Phi_{2}$ is a poset isomorphism and $\left|X_{1}\right|>2$, then the function $h_{i}: X_{1} \rightarrow X_{2}$, such that $h_{i}(x)=J_{\Phi_{2}}\left(i\left(K_{\Phi_{1}}(x)\right)\right)$ for any $x \in X_{1}$, is a $k$-homeomorphism.

Now, let $X$ be a Tychonoff space and let $K(X)$ denote the poset of all Hausdorff compactifications of $X$ (see, e.g., [3]).

For any $c X, d X \in K(X)$ such that $c X<d X$, let

$$
\pi_{d c}=\pi_{d c X}: d X \rightarrow c X
$$

be the canonical map (i.e., it is continuous and $\pi_{d c \mid X}=\operatorname{id}_{X}$ ).

Then $\pi_{d c}^{-1}(c X \backslash X)=d X \backslash X$ and the mapping

$$
r \pi_{d c}=r \pi_{d c X} \stackrel{\text { def }}{=} \pi_{d c}: d X \backslash X \rightarrow c X X
$$

is perfect and onto, that is, $r \pi_{d c} \in \mathscr{P}(d X \backslash X)$.

Fix $e X \in K(X)$ and let

$$
K(e X)=\{c X \in K(X): c X \leq e X\}
$$

(in particular, $K(\beta X)=K(X))$.

In [2], the function

$$
\sigma_{e X}: K(e X) \rightarrow \mathscr{P}(e X \backslash X)
$$

was defined by $\sigma_{e X}(c X)=r \pi_{e c}$ and the following lemma was proved.

LEMMA 2.11. $\sigma_{e X}$ is an isomorphism of the posets $K(e X)$ and

$$
\mathscr{L}(e X) \stackrel{\text { def }}{=} \sigma_{e X}(K(e X)) \text {. }
$$


The following two lemmas were also proved in [2].

LEMMA 2.12. $\mathscr{F} \mathscr{S}(e X \backslash X) \subset \mathscr{L}(e X)$.

LEMMA 2.13. If $X$ is a locally compact space, then $\mathscr{L}(e X)=\mathscr{P}(e X \backslash X)$.

\section{On the homeomorphisms of two pairs of spaces}

Definition 3.1. A space $X$ and a closed subset $A$ are called a pair of spaces and denoted by $(X, A)$.

DEFINITION 3.2. Suppose $(X, A)$ and $(Y, B)$ are pairs of spaces, where $X, Y$ are Hausdorff. Then a homeomorphism (a $k$-homeomorphism) $h: X \rightarrow Y$ is called a homeomorphism (a $k$-homeomorphism) of the pair $(X, A)$ onto the pair $(Y, B)$ if $h(A)=B$.

Let $X$ be a Hausdorff space and $A$ a closed subset of $X$.

For any $\lambda \in \mathscr{P}(X)$, let

$$
\operatorname{res}_{X A}(\lambda)=\lambda: A \longrightarrow \lambda(A)
$$

that is, $\operatorname{res}_{X A}(\lambda)$ is the corestriction of $\lambda$ to $A$.

Evidently, $\operatorname{res}_{X A}(\mathscr{P}(X)) \subset \mathscr{P}(A)$ and $\operatorname{res}_{X A}: \mathscr{P}(X) \rightarrow \mathscr{P}(A)$ is monotonous.

It is not difficult to prove the following lemma.

LEMMA 3.3. $\operatorname{res}_{X A}(\mathscr{P}(X))=\mathscr{P}(A), \operatorname{res}_{X A}(\mathscr{F} \mathscr{S}(X))=\mathscr{F} \mathscr{Y}(A)$, and $\operatorname{res}_{X A}\left(\mathscr{D}(X) \cup\left\{\operatorname{id}_{X}\right\}\right)=$ $\mathscr{D}(A) \cup\left\{\operatorname{id}_{A}\right\}$.

Let $\mathscr{F} \mathscr{Y}(X) \subset \mathscr{I}(X) \subset \mathscr{P}(X)$ and $\mathscr{F} \mathscr{Y}(A) \subset \mathscr{I}(A) \subset \mathscr{P}(A)$. Then, clearly,

$$
\operatorname{res}_{X A}\left(K_{\mathscr{F}(X)}(x)\right) \backslash\left\{\operatorname{id}_{A}\right\}=K_{\mathscr{\Psi}(A)}(x) \quad \forall x \in A .
$$

THEOREM 3.4. Let $X$ and $Y$ be Hausdorff spaces, let $A$ be a closed subset of $X$, and let $B$ be a closed subset of $Y$,

$$
\begin{aligned}
\min \{|X|,|Y|\} \geq 3, & \min \{|A|,|B|\} \geq 2, \\
\mathscr{F} \mathscr{Y}(X) \subset \mathscr{I}(X) \subset \mathscr{P}(X), & \mathscr{F} \mathscr{Y}(A) \subset \mathscr{I}(A) \subset \mathscr{P}(A), \\
\mathscr{F} \mathscr{Y}(Y) \subset \mathscr{I}(Y) \subset \mathscr{P}(Y), & \mathscr{F} \mathscr{S}(B) \subset \mathscr{I}(B) \subset \mathscr{P}(B),
\end{aligned}
$$

and let

$$
i_{X Y}: \mathscr{I}(X) \longrightarrow \mathscr{I}(Y), \quad i_{A B}: \mathscr{I}(A) \longrightarrow \mathscr{I}(B)
$$

be poset isomorphisms such that

$$
i_{A B} \circ \operatorname{res}_{X A}=\operatorname{res}_{Y B} \circ i_{X Y} .
$$

Then

(i) in the case $\min \{|A|,|B|\} \geq 3$ for $k$-homeomorphisms $h_{i_{A B}}: A \rightarrow B$ and $h_{i_{X Y}}: X \rightarrow Y$ (see Theorem 2.10),

$$
h_{i_{A B}}(x)=h_{i_{X Y}}(x) \quad \text { for every } x \in A
$$


(ii) in the case $\min \{|A|,|B|\}=2$, there exists a homeomorphism $h_{i_{A B}}(x): A \rightarrow B$ such that (3.6) is also true.

Thus

$$
h_{i_{X Y}}(A) \subset B, \quad h_{i_{A B}}=h_{i_{X Y}}: A \longrightarrow B,
$$

and so $h_{i_{X Y}}$ is a $k$-homeomorphism of $(X, A)$ onto $(Y, B)$.

If, additionally, $X$ and $Y$ are $k$-spaces, then $h_{i_{X Y}}$ and $h_{i_{A B}}$ are homeomorphisms, and so $h_{i_{X Y}}$ is a homeomorphism of $(X, A)$ onto $(Y, B)$.

Proof. First, let $\min \{|A|,|B|\} \geq 3$.

Let $x \in A$. Then, by Theorem 2.10, $h_{i_{X Y}}$ and $h_{i_{A B}}$ are $k$-homeomorphisms and

$$
\begin{aligned}
h_{i_{A B}}(x) & =J_{\mathscr{F}(B)}\left(i_{A B}\left(K_{\mathscr{I}(A)}(x)\right)\right) \quad(\text { by }(3.2)) \\
& =J_{\mathscr{I}(B)}\left(i_{A B}\left(\operatorname{res}_{X A}\left(K_{\mathscr{I}(X)}(x)\right) \backslash\left\{\operatorname{id}_{A}\right\}\right)\right)
\end{aligned}
$$

(by (3.5) and since $i_{A B}$ is a poset isomorphism)

$$
=J_{\mathscr{Y}(B)}\left(\operatorname{res}_{Y B}\left(i_{X Y}\left(K_{\mathscr{\Psi}(X)}(x)\right)\right) \backslash\left\{\operatorname{id}_{B}\right\}\right)
$$

(by (2.2) and since $i_{X Y}$ is a poset isomorphism)

$$
=J_{\mathscr{F}(B)}\left(\operatorname{res}_{Y B}\left(K_{\mathscr{Y}(Y)}\left(J_{\mathscr{F}(Y)}\left(i_{X Y}\left(K_{\mathscr{Y}(X)}(x)\right)\right)\right)\right) \backslash\left\{\operatorname{id}_{B}\right\}\right)
$$

(by the definition of $h_{i_{X Y}}$ )

$$
\begin{aligned}
& =J_{\mathscr{I}(B)}\left(\operatorname{res}_{Y B}\left(K_{\mathscr{I}(Y)}\left(h_{i_{X Y}}(x)\right)\right) \backslash\left\{\operatorname{id}_{B}\right\}\right) \\
& =J_{\mathscr{I}(B)}\left(K_{\mathscr{I}(B)}\left(h_{i_{X Y}}(x)\right)\right) \quad(\text { by }(2.1)) \\
& =h_{i_{X Y}}(x) .
\end{aligned}
$$

Now, let $|A|=2$. Since $i_{A B}$ is a poset isomorphism, $|\mathscr{F}(B)|=|\mathscr{T}(A)|=2$ and so $|B|=2$.

There is a unique dual point $\lambda \in \mathscr{I}(X)$ such that $\lambda^{-1}\left(\left\{t_{\lambda}\right\}\right)=A$. Let $A=\left\{x_{1}, x_{2}\right\}$. Evidently, $K_{\mathscr{I}(X)}\left(x_{1}\right) \cap K_{\mathscr{I}(X)}\left(x_{2}\right)=\{\lambda\}$. Then, by (2.2),

$$
\begin{aligned}
K_{\mathscr{Y}(Y)}\left(h_{i_{X Y}}\left(x_{i}\right)\right) & =K_{\mathscr{F}(Y)}\left(J_{\mathscr{\Psi}(Y)}\left(i_{X Y}\left(K_{\mathscr{\Psi}(X)}\left(x_{i}\right)\right)\right)\right) \\
& =i_{X Y}\left(K_{\mathscr{\Psi}(X)}\left(x_{i}\right)\right) \text { for } i=1,2 .
\end{aligned}
$$

Hence

$$
\begin{aligned}
i_{X Y}(\{\lambda\}) & =i_{X Y}\left(K_{\mathscr{I}(X)}\left(x_{1}\right) \cap K_{\mathscr{I}(X)}\left(x_{2}\right)\right) \\
& =i_{X Y}\left(K_{\mathscr{F}(X)}\left(x_{1}\right)\right) \cap i_{X Y}\left(K_{\mathscr{\Psi}(X)}\left(x_{2}\right)\right) \\
& =K_{\mathscr{I}(Y)}\left(h_{i_{X Y}}\left(x_{1}\right)\right) \cap K_{\mathscr{f}(Y)}\left(h_{i_{X Y}}\left(x_{2}\right)\right) .
\end{aligned}
$$

Thus, for $\eta=i_{X Y}(\lambda), \eta^{-1}\left(\left\{t_{\eta}\right\}\right)=\left\{h_{i_{X Y}}\left(x_{1}\right), h_{i_{X Y}}\left(x_{1}\right)\right\}=h_{i_{X Y}}(A)$. But, by (3.5), $\xi=$ $\operatorname{res}_{Y B}(\eta)=\operatorname{res}_{Y B}\left(i_{X Y}(\lambda)\right)=i_{A B}\left(\operatorname{res}_{X A}(\lambda)\right)$. Since $\operatorname{res}_{X A}(\lambda)$ is a dual point in $\mathscr{I}(A)$ and $i_{A B}$ is a poset isomorphism, $\xi$ is also a dual point in $\mathscr{\Psi}(B)$. Thus, $B=\xi^{-1}\left(\left\{t_{\xi}\right\}\right)=\eta^{-1}\left(\left\{t_{\eta}\right\}\right)=$ $h_{i_{X Y}}(A)$.

\section{Extensions of Magill's and Rayburn's theorems to mappings}

DEFINITION 4.1. For mappings $f_{j}: X_{j} \rightarrow Y$ of (Hausdorff) spaces $X_{j}$ (with $j=1,2$ ), a ( $k$-) homeomorphism $h: X_{1} \rightarrow X_{2}$ is called a $(k-)$ homeomorphism of $f_{1}$ onto $f_{2}$ 
if $f_{1}=f_{2} \circ h$. The mappings $f_{j}: X_{j} \rightarrow Y$ (for $\left.j=1,2\right)$ are $(k-)$ homeomorphic if there exists a ( $k$-) homeomorphism of $f_{1}$ onto $f_{2}$.

It is not difficult to prove the following lemma.

LEMMA 4.2. For mappings $f_{j}: X_{j} \rightarrow Y$ (with $\left.j=1,2\right)$ and for ( $k$-) homeomorphism $h: X_{1} \rightarrow X_{2}$ of spaces $X_{1}$ and $X_{2}$, the following conditions are equivalent:

(i) $h$ is a homeomorphism of $f_{1}$ onto $f_{2}$;

(ii) $h\left(f_{1}^{-1}(\{y\})\right) \subset f_{2}^{-1}(\{y\})$ for every $y \in Y$;

(iii) there is a function $h_{y}: f_{1}^{-1}(\{y\}) \rightarrow f_{2}^{-1}(\{y\})$ such that $h_{y}=h: f_{1}^{-1}(\{y\}) \rightarrow$ $f_{2}^{-1}(\{y\})$ for every $y \in Y$.

Given a Tychonoff space $X$ and a closed subset $A$ of $X$, we may define a function

$$
\operatorname{kres}_{X A}: K(X) \longrightarrow K(A)
$$

such that

$$
\operatorname{kres}_{X A}(c X)=c l_{c X}(A) \quad \text { for every } c X \in K(X) \text {. }
$$

If $e X, c X \in K(X)$ and $c X<e X$, then $\pi_{e c X}\left(c l_{e X}(A)\right)=c l_{c X}(A), \pi_{e c X}(A)=A$, and $\left(\pi_{e c X}: A \rightarrow A\right)=\operatorname{id}_{A}$.

Consequently, $\left(e A=\operatorname{kres}_{X A}(e X)\right)>\left(c A=\operatorname{kres}_{X A}(c X)\right)$ and $\pi_{e c A}=\pi_{e c X}: e A \rightarrow$ $c A$. Thus, $\operatorname{kres}_{X A}$ is monotone and $r \pi_{e c A}=r \pi_{e c X}: e A \backslash A \rightarrow c A \backslash A$, that is, $r \pi_{e c A}=$ $\operatorname{res}_{e X \backslash X, e A \backslash A}\left(r \pi_{e c X}\right)$. It follows from this that $\left(\sigma_{e A} \circ \operatorname{kres}_{X A}\right)(c X)=\sigma_{e A}(c A)=r \pi_{e c A}=$ $\operatorname{res}_{e X \backslash X, e A \backslash A}\left(r \pi_{e c X}\right)=\operatorname{res}_{e X \backslash X, e A \backslash A}\left(\sigma_{e X}(c X)\right)$.

We have then proven the following lemma.

LEMMA 4.3. If $e X \in K(X)$ and $e A=\operatorname{kres}_{X A}(e X)$, then

$$
\operatorname{res}_{e X \backslash X, e A \backslash A} \circ \sigma_{e X}=\left.\sigma_{e A} \circ \operatorname{kres}_{X A}\right|_{K(e X)} .
$$

Let $f: X \rightarrow Y$ be a mapping to a Tychonoff space $Y$ and let $\beta f^{+}: \beta X \rightarrow \beta Y$ be the (usual) continuous extension of $f$ over the Stone-Cěch compactifications $\beta X, \beta_{f} X=$ $\left(\beta f^{+}\right)^{-1}(Y)$, and $\beta f=\beta f^{+}: \beta_{f} X \rightarrow Y$. Evidently, the mapping $\beta f$ is perfect. We note that $X$ is $C^{*}$-embedded in $\beta_{f} X$ because $X \subset \beta_{f} X \subset \beta X$. Recall that $f$ is called a $W Z$ mapping [6] (resp., a Z-mapping) if $(\beta f)^{-1}(\{y\})=c l_{\beta_{f} X}\left(f^{-1}(\{y\})\right)$ for every $y \in$ $Y$ (resp., if $f(Z)$ is closed for any zero-set $Z$ in $X$ ). It is clear that $(\beta f)^{-1}(\{y\})=$ $\beta\left(f^{-1}(\{y\})\right)$ if the space $X$ is normal and $f$ is a WZ-mapping. It is known [6] that every Z-mapping is a WZ-mapping.

THEOREM 4.4. Let $X_{j}$ be a Tychonoff space, let $Y$ be a compact Hausdorff space, let $f_{j}: X_{j} \rightarrow Y$ be a WZ-mapping, let $e X_{j}$ be a Hausdorff compactification of $X_{j}$, and let $e f_{j}: e X_{j} \rightarrow Y$ be a continuous extension of $f_{j}$ (thus, $e f_{j}$ is a compactification of $f_{j}$ ) for $j=1,2$. Let also $X_{j y}=f_{j}^{-1}(\{y\}), e X_{j y}=c l_{e X_{j}}\left(X_{j y}\right)$ (i.e., $e X_{j y}=\operatorname{kres}_{X_{j} X_{j y}}\left(e X_{j}\right)$ ) for $j=1,2$, and suppose that there exist poset isomorphisms $i: K\left(e X_{1}\right) \rightarrow K\left(e X_{2}\right)$ and $i_{y}: K\left(e X_{1 y}\right) \rightarrow K\left(e X_{2 y}\right)$ such that

$$
i_{y} \circ \operatorname{kres}_{X_{1} X_{1 y}}=\operatorname{kres}_{X_{2} X_{2 y}} \circ i \text { for every } y \in Y \text {. }
$$


Then the remainders $e f_{j} \backslash f_{j} \stackrel{\text { def }}{=} e f_{j}: e X_{j} \backslash X_{j} \rightarrow Y$ of $e f_{j}$ for $j=1,2$ are $k$-homeomorphic (more exactly, if $\min \left\{\left|e X_{1} \backslash X_{1}\right|,\left|e X_{2} \backslash X_{2}\right|\right\} \geq 3$, then the function $h_{\sigma_{e X_{2}} \circ i \circ \sigma_{e X_{1}}^{-1}}: e X_{1} \backslash X_{1} \rightarrow$ $e X_{2} \backslash X_{2}$ is a $k$-homeomorphism of e $f_{1} \backslash f_{1}$ onto $\left.e f_{2} \backslash f_{2}\right)$. If, additionally, $X_{1}$ and $X_{2}$ are $k$-absolute spaces, then the remainders $e f_{1} \backslash f_{1}$ and $e f_{2} \backslash f_{2}$ are homeomorphic.

Proof. Fix $y \in Y$. Let $R_{e j}=e X_{j} \backslash X_{j}$ and $R_{e j y}=e X_{j y} \backslash X_{j y}$ for $j=1,2$.

By Lemmas 2.11 and 2.12, the mappings $\sigma_{e X_{j}}: K\left(e X_{j}\right) \rightarrow\left(\Phi_{j} \stackrel{\text { def }}{=} \sigma_{e X_{j}}\left(K\left(e X_{j}\right)\right)\right)$ and $\sigma_{e X_{j y}}: K\left(e X_{j y}\right) \rightarrow\left(\mathscr{I}_{j y} \stackrel{\text { def }}{=} \sigma_{e X_{j y}}\left(K\left(e X_{j y}\right)\right)\right)$ are poset isomorphisms and $\mathscr{F} \mathscr{Y}\left(R_{e j}\right) \subset \mathscr{I}_{j}$, $\mathscr{F} \mathscr{Y}\left(R_{e j y}\right) \subset \mathscr{I}_{j y}$ for $j=1,2$.

Hence, $i_{12}=\sigma_{e X_{2}} \circ i \circ \sigma_{e X_{1}}^{-1}: \mathscr{I}_{1} \rightarrow \Phi_{2}$ and $i_{12 y}=\sigma_{e X_{2 y}} \circ i_{y} \circ \sigma_{e X_{1 y}}^{-1}: \mathscr{I}_{1 y} \rightarrow \mathscr{I}_{2 y}$ are poset isomorphisms too.

We consider the following diagram:

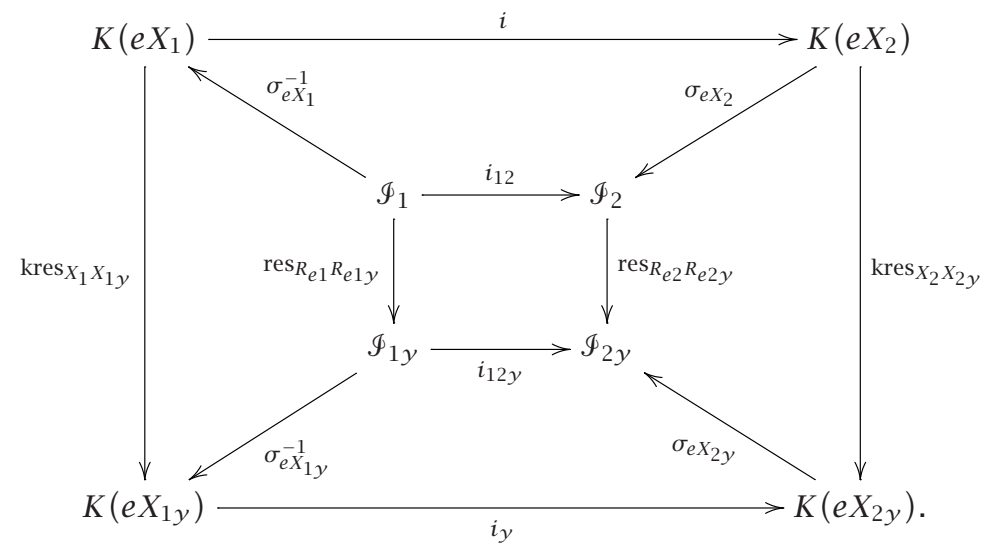

By (4.4), the external part of it is commutative. We prove that its internal part is commutative too, that is, that

$$
\operatorname{res}_{R_{e 2} R_{e 2 y}} \circ i_{12}=i_{12 y} \circ \operatorname{res}_{R_{e 1} R_{e 1 y}} .
$$

By (4.3), for $j=1,2$, we have (see the diagram)

$$
\operatorname{res}_{R_{e j} R_{e j y}} \circ \sigma_{e X_{j}}=\left.\sigma_{e X_{j y}} \circ \operatorname{kres}_{X_{j} X_{j y}}\right|_{K\left(e X_{j}\right)} .
$$

Thus, since $\sigma_{e X_{j}}, \sigma_{e X_{j y}}$ are isomorphisms,

$$
\begin{gathered}
\left.\operatorname{kres}_{X_{j} X_{j y}}\right|_{K\left(e X_{j}\right)} \circ \sigma_{e X_{j}}^{-1}=\sigma_{e X_{j y}}^{-1} \circ \operatorname{res}_{R_{e j} R_{e j y}}, \\
\operatorname{res}_{R_{e j} R_{e j y}}\left(\Phi_{j}\right) \subset \Phi_{j y} .
\end{gathered}
$$


Hence,

$$
\begin{aligned}
\operatorname{res}_{R_{e 2} R_{e 2 y}} \circ i_{12} & =\operatorname{res}_{R_{e 2} R_{e 2 y}} \circ \sigma_{e X_{2}} \circ i \circ \sigma_{e X_{1}}^{-1} \\
& =\sigma_{e X_{2 y}} \circ \operatorname{kres}_{X_{2} X_{2 y}} \circ i \circ \sigma_{e X_{1}}^{-1} \\
& =\sigma_{e X_{2 y}} \circ i_{y} \circ \operatorname{kres}_{X_{1} X_{1 y}} \circ \sigma_{e X_{1}}^{-1} \\
& =\sigma_{e X_{2 y}} \circ i_{y} \circ \sigma_{e X_{1 y}}^{-1} \circ \operatorname{res}_{R_{e 1} R_{e 1 y}} \\
& =i_{12 y} \circ \operatorname{res}_{R_{e 1} R_{e 1 y}} .
\end{aligned}
$$

By Theorem 3.4, in the case $\min \left\{\left|R_{e 1}\right|,\left|R_{e 2}\right|,\left|R_{e 1}\right|,\left|R_{e 2 y}\right|\right\} \geq 3$, the homeomorphisms $h_{i_{12}}: R_{e 1} \rightarrow R_{e 2}$ and $h_{i_{12 y}}: R_{e 1 y} \rightarrow R_{e 2 y}$ are such that

$$
h_{i_{12 y}}=h_{i_{12}}: R_{e 1 y} \rightarrow R_{e 2 y}
$$

and in the case $\min \left\{\left|R_{e 1}\right|,\left|R_{e 2}\right|\right\} \geq 3, \min \left\{\left|R_{e 1}\right|,\left|R_{e 2 y}\right|\right\} \geq 2$, there exists a homeomorphism $h_{i_{12 y}}: R_{e 1 y} \rightarrow R_{e 2 y}$ such that (4.10) is also true.

Finally, in the case $\min \left\{\left|R_{e 1}\right|,\left|R_{e 2}\right|\right\} \geq 3, \min \left\{\left|R_{e 1}\right|,\left|R_{e 2 y}\right|\right\} \leq 1$, the existence of a homeomorphism $h_{i_{12 y}}: R_{e 1 y} \rightarrow R_{e 2 y}$ such that (4.10) holds is evident.

Thus, in the case $\min \left\{\left|R_{e 1}\right|,\left|R_{e 2}\right|\right\} \geq 3$, (4.10) holds for every $y \in Y$.

In the case $\min \left\{\left|R_{e 1}\right|,\left|R_{e 2}\right|\right\} \leq 2$, the existence of homeomorphisms $h_{i_{12}}: R_{e 1} \rightarrow R_{e 2}$ and $h_{i_{12 y}}: R_{e 1 y} \rightarrow R_{e 2 y}$ such that (4.10) holds is evident.

Since $f_{j}$ is a WZ-mapping, $R_{e j y}=\left(e f_{j}\right)^{-1}(\{y\}) \backslash f_{j}^{-1}(\{y\})$ for $j=1,2$, and so

$$
h_{i_{12 y}}=h_{i_{12}}:\left(e f_{1}\right)^{-1}(\{y\}) \backslash f_{1}^{-1}(\{y\}) \longrightarrow\left(e f_{2}\right)^{-1}(\{y\}) \backslash f_{2}^{-1}(\{y\}) \quad \text { for every } y \in Y
$$

By Lemma $4.2, h_{i_{12}}$ is a $k$-homeomorphism of $e f_{1} \backslash f_{1}$ onto $e f_{2} \backslash f_{2}$.

COROLLARY 4.5 [2]. Let $X_{1}, X_{2}$ be Tychonoff spaces and let e $X_{1}, e X_{2}$ be their Hausdorff compactifications. If $K\left(e X_{1}\right)$ and $K\left(e X_{2}\right)$ are poset isomorphic, then the remainders e $X_{1} \backslash$ $X_{1}$ and $e X_{2} \backslash X_{2}$ are $k$-homeomorphic, and they are homeomorphic if, additionally, $X_{1}$, $X_{2}$ are $k$-absolute spaces.

Proof. It is sufficient to apply Theorem 4.4 to (the simplest) mappings $f_{j}$ of $X_{j}$ to the single point space $Y$ for $j=1,2$.

In particular, in Corollary 4.5, for $e X_{j}=\beta X_{j}(j=1,2)$, we have Rayburn's Theorem 1.3. Thus, Theorem 4.4 is a generalization of this theorem to mappings.

THEOREM 4.6. Let $X_{j}$ be a locally compact Tychonoff space, let $Y$ be a compact Hausdorff space, let $f_{j}: X_{j} \rightarrow Y$ be a WZ-mapping, let e $X_{j}$ be a Hausdorff compactification of $X_{j}$, and let $e f_{j}: e X_{j} \rightarrow Y$ be a continuous extension of $f_{j}$ for $j=1,2$. Let also $X_{j y}=f_{j}^{-1}(\{y\}), e X_{j y}=c l_{e X_{j}}\left(X_{j y}\right)$ (i.e., $\left.e X_{j y}=\operatorname{kres}_{X_{j} X_{j y}}\left(e X_{j}\right)\right)($ for $j=1,2)$. Then the remainders $e f_{j} \backslash f_{j}=e f_{j}: e X_{j} \backslash X_{j} \rightarrow Y$ of $e f_{j}$ for $j=1,2$ are homeomorphic if and only 
if there exist poset isomorphisms $i: K\left(e X_{1}\right) \rightarrow K\left(e X_{2}\right)$ and $i_{y}: K\left(e X_{1 y}\right) \rightarrow K\left(e X_{2 y}\right)$ such that (4.4) holds.

Proof. One half of the theorem follows from Theorem 4.4.

Let $R_{e j}=e X_{j} \backslash X_{j}$ and $R_{e j y}=e X_{j y} \backslash X_{j y}$ for all $y \in Y$ and $j=1,2$. Now, suppose that the remainders $e f_{1} \backslash f_{1}$ and $e f_{2} \backslash f_{2}$ are homeomorphic. Then there exists a homeomorphism $h: R_{e 2} \rightarrow R_{e 1}$ such that $e f_{2} \backslash f_{2}=\left(e f_{1} \backslash f_{1}\right) \circ h$. Hence, the mappings $h_{y}=h: R_{e 2 y} \rightarrow R_{e 1 y}$ are homeomorphisms for all $y \in Y$. Since $f_{j}$ is a WZ-mapping, $\left(e f_{j}\right)^{-1}(\{y\})$ is a compactification of $f_{j}^{-1}(\{y\})$, and so $e X_{j y}=\left(e f_{j}\right)^{-1}(\{y\})$ for all $y \in Y$ and $j=1$,2. Evidently, $i_{12}: \mathscr{P}\left(R_{e 1}\right) \rightarrow \mathscr{P}\left(R_{e 2}\right)$ and $i_{12 y}: \mathscr{P}\left(R_{e 1 y}\right) \rightarrow \mathscr{P}\left(R_{e 2 y}\right)$, such that $i_{12}(\lambda)=\lambda \circ h$ for $\lambda \in \mathscr{P}\left(X_{1}\right)$ and $i_{12 y}(\lambda)=\lambda \circ h_{y}$ for $\lambda \in \mathscr{P}\left(X_{1 y}\right)$ and all $y \in Y$, are poset isomorphisms.

We prove that (4.4) holds for all $y \in Y$.

Indeed, for every $\lambda \in \mathscr{P}\left(R_{e 1}\right)$ and $y \in Y$, we have

$$
\begin{aligned}
i_{12 y} \circ \operatorname{res}_{R_{e 1} R_{e 1 y}}(\lambda) & =i_{12 y}\left(\left.\lambda\right|_{R_{e 1 y}}\right)=\left.\lambda\right|_{R_{e 1}} \circ h_{y} \\
& =\left.(\lambda \circ h)\right|_{R_{e 2 y}}=\left.\left(i_{12}(\lambda)\right)\right|_{R_{e 2 y}} \\
& =\operatorname{res}_{R_{e 2} R_{e 2 y}} \circ i_{12}(\lambda) .
\end{aligned}
$$

By Lemmas 2.11 and 2.13, $\sigma_{e X_{j}}: K\left(e X_{j}\right) \rightarrow \mathscr{P}\left(R_{e j}\right)$ and $\sigma_{e X_{j y}}: K\left(e X_{j y}\right) \rightarrow \mathscr{P}\left(R_{e j y}\right)$, for $j=1,2$ and $y \in Y$, are poset isomorphisms. Hence, $i=\sigma_{e X_{2}}^{-1} \circ i_{12} \circ \sigma_{e X_{1}}$ and $i_{y}=\sigma_{e X_{2 y}}^{-1} \circ$ $i_{12 y} \circ \sigma_{e X_{1 y}}$ are poset isomorphisms for $y \in Y$ and $j=1,2$.

We consider the diagram obtained from the previous one by replacing $\mathscr{I}_{j}$ and $\mathscr{I}_{j y}$ by $\mathscr{P}\left(R_{e j}\right)$ and $\mathscr{P}\left(R_{e j y}\right)$ for $j=1,2$ and $\sigma_{e X_{1}}^{-1}, \sigma_{e X_{1 y}}^{-1}, \sigma_{e X_{2}}, \sigma_{e X_{2 y}}$ by $\sigma_{e X_{1}}, \sigma_{e X_{1 y}}, \sigma_{e X_{2}}^{-1}$, $\sigma_{e X_{2 y}}^{-1}$, respectively. By (4.12), its internal part is commutative. As above, in the proof of Theorem 4.4, we can prove that its external part is commutative too, that is, that (4.4) holds.

COROLlaRY 4.7 [2]. Let $X_{1}, X_{2}$ be locally compact Tychonoff spaces and let $e X_{1}, e X_{2}$ be Hausdorff compactifications of $X_{1}$ and $X_{2}$, respectively. Then the remainders e $X_{1} \backslash X_{1}$ and $e X_{2} \backslash X_{2}$ are homeomorphic if and only if $K\left(e X_{1}\right)$ and $K\left(e X_{2}\right)$ are poset isomorphic.

Proof. It is sufficient to apply Theorem 4.6 to the simplest mappings $f_{j}$ of $X_{j}$ and $e f_{j}$ of $e X_{j}$ to the single point space $Y$ for $j=1,2$.

In particular, when in Corollary $4.7, e X_{j}=\beta X_{j}$ for $j=1$, 2, we have Magill's theorem from [8]. Thus, Theorem 4.6 is a generalization of this theorem to mappings.

5. Reformulations of results obtained above and some examples. Some readers may find that Theorems 4.4 and 4.6 do not sound very natural. The reformulations, in the framework of FGT, sound better to us.

We will start with some definitions and results of FGT.

A mapping is called compact if it is perfect.

The following is evident. 
LEMMA 5.1. For a compact Hausdorff space $Y$, a mapping $f: X \rightarrow Y$ is compact if and only if $X$ is compact.

Definition 5.2. A mapping $f: X \rightarrow Y$ is said to be $\mathrm{T}_{0}$ [11] if for every $x, x^{\prime} \in X$ such that $x \neq x^{\prime}$ and $f(x)=f\left(x^{\prime}\right)$, there exists a neighbourhood of $x$ in $X$ which does not contain $x^{\prime}$ or a neighbourhood of $x^{\prime}$ in $X$ not containing $x$.

DEFINITION 5.3. A mapping $f: X \rightarrow Y$ is said to be completely regular [11] if for every closed set $F$ of $X$ and $x \in X \backslash F$, there exist a neighbourhood $O$ of $f(x)$ in $Y$ and a continuous function $\varphi: f^{-1}(O) \rightarrow[0,1]$ such that $\varphi(x)=0$ and $\varphi\left(F \cap f^{-1}(O)\right) \subseteq\{1\}$. A completely regular $T_{0}$-mapping is called Tychonoff (or $\left.\mathrm{T}_{3(1 / 2)}\right)$.

It is not difficult to prove the following lemma.

LEMMA 5.4 [11]. For a Tychonoff space $Y$, a mapping $f: X \rightarrow Y$ is Tychonoff if and only if $X$ is Tychonoff.

DeFinition 5.5. A compact Tychonoff mapping ef $: e_{f} X \rightarrow Y$ is called a Tychonoff compactification of a Tychonoff mapping $f: X \rightarrow Y$ if $X \subset e_{f} X, X$ is dense in $e_{f} X$, and $\left.e_{f} X\right|_{X}=f$ (more precisely, if some embedding $e: X \rightarrow e_{f} X$ is fixed so that $e(X)$ is dense in $e_{f} X$ and $f=e f \circ e$, but usually, $X$ and $e(X)$ are identified by means of $e$ ).

Throughout the rest of the paper, we fix a space $Y$ and we will consider only Tychonoff mappings to $Y$ and their Tychonoff compactifications.

DEFINITION 5.6. A mapping $\lambda: d_{f} X \rightarrow c_{f} X$ between two compactifications $c f$ : $c_{f} X \rightarrow Y$ and $d f: d_{f} X \rightarrow Y$ is called canonical if $d f=c f \circ \lambda$ and $\lambda_{\mid X}=\operatorname{id}_{X}$. In this case, one says that we have a canonical morphism $\lambda: d f \rightarrow c f$ (and we write that $d f>c f)$.

It is not difficult to prove that $d f$ and $c f$ are homeomorphic if and only if $d f>c f$ and $c f>d f$ (see, e.g., [1]).

It is proved in [11] (see also [1]) that all compactifications of a mapping to $Y$ form a set up to canonical homeomorphisms. This set will be denoted by $T K(f)$. Evidently, with respect to the just defined relation $>, T K(f)$ is a poset.

In [11], it is also proved that there exists the maximal element $\beta f: \beta_{f} X \rightarrow Y$ in $T K(f)$ and that, if $Y$ is Tychonoff, $\beta f$ may be obtained in the following way.

By Lemma 5.4, $X$ is Tychonoff. Hence, there exists the unique continuous extension $\beta f^{+}: \beta X \rightarrow \beta Y$ of $f$. Then $\beta_{f} X=\left(\beta f^{+}\right)^{-1}(Y)$ and $\beta f=\beta f^{+}: \beta_{f} X \rightarrow Y$.

For a compactification ef $: e_{f} X \rightarrow Y$ of a mapping $f: X \rightarrow Y$, the mapping ef $\backslash f=$ ef $: e_{f} X \backslash X \rightarrow Y$ is called the remainder of $e f$.

A mapping $f: X \rightarrow Y$ is called locally compact [7] if for any point $x \in X$, there exists a neighbourhood $O$ of $x$ in $X$ such that $\left.f\right|_{c l_{X}(O)}: c l_{X}(O) \rightarrow Y$ is compact.

It is not difficult to prove that

(i) for a locally compact Tychonoff space $Y$, a mapping $f: X \rightarrow Y$ is locally compact if and only if $X$ is locally compact; 
(ii) a mapping $f: X \rightarrow Y$ is locally compact if and only if $X$ is open in $\beta_{f} X$ or, equivalently, if $X$ is open in $e_{f} X$ for any compactification $e f: e_{f} X \rightarrow Y$ of $f$.

Now, Theorem 4.4 can be reformulated in the following way.

THEOREM 5.7. Let $Y$ be a compact Hausdorff space and $e f_{j}: e_{f_{j}} X_{j} \rightarrow Y$ a Tychonoff compactification of a locally compact Tychonoff WZ-mapping $f_{j}: X_{j} \rightarrow Y$ (for $j=1,2$ ). Let also $X_{j y}=f_{j}^{-1}(\{y\}), e X_{j y}=c l_{e X_{j}}\left(X_{j y}\right)$ for $j=1,2$. Then the remainders $e f_{1} \backslash f_{1}$ and $e f_{2} \backslash f_{2}$ are homeomorphic if and only if there exist poset isomorphisms $i: K\left(e_{f_{1}} X_{1}\right) \rightarrow$ $K\left(e_{f_{2}} X_{2}\right)$ and $i_{y}: K\left(e X_{1 y}\right) \rightarrow K\left(e X_{2 y}\right)$ such that (4.4) holds.

Theorem 3.4 may be reformulated analogously. But, even in the style of Theorem 5.7, the formulation of Theorem 4.4 (and Theorem 3.4) seems too complicated, but this complexity may not be avoided. In order to explain why, for any fixed space $Y$, consider the category $\operatorname{Top}_{Y}$, where

$$
\mathrm{Ob}\left(\operatorname{Top}_{Y}\right)=\{f \in C(X, Y): X \in \mathrm{Ob}(\mathbf{T o p})\}
$$

is the class of the objects and, for every pair $f: X \rightarrow Y, g: Z \rightarrow Y$ of objects,

$$
M(f, g)=\{\lambda \in C(X, Z): g \circ \lambda=f\}
$$

is the class of the morphisms from $f$ to $g$, whose generic representation is denoted in short by $\lambda: f \rightarrow g$.

So, the question is: may the passage from the category Top to the category Top $_{Y}$ allow us to give simpler variants of Theorems 4.4 and 4.6 which can generalize Magill's and Rayburn's theorems?

In this connection, we will give two examples which demonstrate that in the framework of $\operatorname{Top}_{Y}$, such generalizations are impossible.

EXAMPLE 5.8. Let $Y=[0,2], X_{1}=X_{2}=\mathbb{N}$, and $f_{j}: X_{j} \rightarrow Y$ be such that $f_{j}\left(X_{j}\right)=\{j\}$ for $j=1$, 2. Then $\beta_{f_{j}} X_{j}=\beta \mathbb{N}, \beta f_{j}\left(\beta_{f_{j}} X_{j}\right)=\{j\}, \beta f_{j} \backslash f_{j}=\beta f_{j}: \beta_{f_{j}} X_{j} \backslash X_{j} \rightarrow Y$, and so $\beta f_{j} \backslash f_{j}=\beta f_{j}: \beta \mathbb{N} \backslash \mathbb{N} \rightarrow Y$, with $\left(\beta f_{j} \backslash f_{j}\right)(\beta \mathbb{N} \backslash \mathbb{N})=\{j\}$ for $j=1,2$. Thus, the remainders $\beta f_{1} \backslash f_{1}$ and $\beta f_{2} \backslash f_{2}$ are not homeomorphic, but $T K\left(f_{1}\right)$ and $T K\left(f_{2}\right)$ are poset isomorphic because they, in fact, coincide with $K(\mathbb{N})$.

This example shows that an extension of Magill's Theorem to the category Top $_{Y}$ must take into consideration fibres of objects of $\operatorname{Top}_{Y}$.

EXAMPLE 5.9. Let $I=\left\{(x, 0) \in \mathbb{R}^{2}: 0 \leq x \leq 1\right\}, L=\left\{(0, y) \in \mathbb{R}^{2}:-1 \leq y \leq 1\right\}, J=$ $\left\{(x, 1) \in \mathbb{R}^{2}: 0 \leq x \leq 1\right\}, Y=I, S_{1}=I \cup L$, and $S_{2}=J \cup L$. Let $j=1,2$. Put $p r_{j}(x, y)=x$ for any $(x, y) \in S_{j}$. Let $\omega_{1}$ be the space of all finite and countable ordinal numbers. Then $\beta \omega_{1}=\omega_{1}+1$. Put $X_{j}=S_{j} \times \omega_{1}$ and let $\pi_{j}$ be the projection of the product $X_{j}$ onto its factor $S_{j}$. Take $f_{j}=p r_{j} \circ \pi_{j}$. Evidently, $f_{j}$ is either closed or open and all its fibres are countable compact. Then $\beta X_{j}=S_{j} \times\left(\omega_{1}+1\right)$ and $R_{j}=\beta X_{j} \backslash X_{j}$ is homeomorphic to $S_{j}$. Let $p_{j}$ be the projection of the product $\beta X_{j}$ onto its factor $S_{j}$. Then $\beta f_{j}=p r_{j} \circ p_{j}$ and $\beta f_{j} \backslash f_{j}=\beta f_{j}: R_{j} \rightarrow Y$ is homeomorphic to $p r_{j}$.

Thus, the remainders $\beta f_{1} \backslash f_{1}$ and $\beta f_{2} \backslash f_{2}$ are not homeomorphic. 
For any $t=(x, 0) \in Y,\left(\beta f_{j}\right)^{-1}(\{t\})=\beta\left(f_{j}^{-1}(\{t\})\right)$, and, for any $t=(x, 0) \in Y$ with $x>0$, the remainders $R_{j t}=\left(\beta f_{j}\right)^{-1}(\{t\}) \backslash f_{j}^{-1}(\{t\})=\beta\left(f_{j}^{-1}(\{t\})\right) \backslash f_{j}^{-1}(\{t\})$ are single points. Hence, $K\left(f_{j}^{-1}(\{t\})\right)$ consists of only one element for $t=(x, 0) \in Y$ with $x>0$. For $t=(0,0)$, the remainder $R_{j t}$ is homeomorphic to $L$. Evidently, $T K\left(f_{j}\right)$ and $K\left(f_{j}^{-1}(\{(0,0)\})\right)$ are poset isomorphic to $\mathscr{P}(L)$.

Now, it is evident that there exist poset isomorphisms $i: T K\left(f_{1}\right) \rightarrow T K\left(f_{2}\right), i_{t}$ : $K\left(f_{1}^{-1}(\{t\})\right) \rightarrow K\left(f_{2}^{-1}(\{t\})\right)$ for $t \in Y$ and monotone functions $m_{t j}: T K\left(f_{j}\right) \rightarrow$ $K\left(f_{j}^{-1}(\{t\})\right)$ for $t \in Y$ and $j=1,2$ such that

(i) for any $c f_{j}: c_{f_{j}} X_{j} \rightarrow Y$ with $c f_{j} \in T K\left(f_{j}\right), m_{t j}\left(c f_{j}\right)=\left(c f_{j}\right)^{-1}(\{t\})$, that is, $m_{t j}\left(c f_{j}\right)$ is the closure of $f_{j}^{-1}(\{t\})$ in $c_{f_{j}} X_{j}$;

(ii) $m_{t 2} \circ i=i_{t} \circ m_{t 1}$ for every $t \in Y$.

Example 5.9 shows that the use of the posets $K\left(e X_{1}\right)$ and $K\left(e X_{2}\right)$ (in particular $K\left(X_{1}\right)$ and $\left.K\left(X_{2}\right)\right)$ instead of $T K\left(f_{1}\right)$ and $T K\left(f_{2}\right)$ is justified.

REMARK 5.10. Let $f: X \rightarrow Y$ be a mapping between Tychonoff spaces $M(f)=$ $\left\{y \in Y: f\right.$ is not closed at $y$ or $f^{-1}(\{y\})$ is not compact $\}$ and $M_{1}(f)=\{y \in Y$ : $\left.\left|(\beta f)^{-1}(\{y\}) \backslash f^{-1}(\{y\})\right|=1\right\}$. Evidently, $M_{1}(f) \subset M(f)$ and $\beta f\left(\beta_{f} X \backslash X\right)=M(f)$. The following proposition is proved in [5].

Proposition 5.11. Let $f: X \rightarrow Y$ and $g: Z \rightarrow T$ be locally compact mappings between Tychonoff spaces and $\left|M_{1}(f)\right|=\left|M_{1}(g)\right|=1$. Then there exists a one-to-one correspondence $\Psi: M(f) \rightarrow M(g)$ such that $(\beta f \backslash f)^{-1}(\{y\})$ and $(\beta g \backslash g)^{-1}(\{\Psi(y)\})$ are homeomorphic for any $y \in Y$. If, additionally, $M(f)$ and $M(g)$ are discrete, then $\beta f \backslash f$ and $\beta g \backslash g$ are homeomorphic in the sense of [5], that is, there exist homeomorphisms $\varphi: \beta_{f} X \backslash X \rightarrow \beta_{g} Z \backslash Z$ and $\psi: M(f) \rightarrow M(g)$ such that $\psi \circ(\beta f \backslash f)=(\beta g \backslash g) \circ \varphi$ if and only if $T K(f)$ and $T K(g)$ are poset isomorphic.

ACKNOWLEDGMENTS. The authors would like to thank the anonymous referees for their very accurate reading and the valuable suggestions. This research was supported by MURST and CNR (GNSAGA). The second author was supported by KCFE and RFFI.

\section{REFERENCES}

[1] I. V. Bludova and G. Nordo, On the posets of all the Hausdorff and all the Tychonoff compactifications of mappings, Questions Answers Gen. Topology 17 (1999), no. 1, 47-55.

[2] I. V. Bludova, G. Nordo, and B. A. Pasynkov, On the homeomorphism of spaces and Magilltype theorems, Questions Answers Gen. Topology 19 (2001), no. 1, 95-105.

[3] R. E. Chandler, Hausdorff Compactifications, Lecture Notes in Pure and Applied Mathematics, vol. 23, Marcel Dekker, New York, 1976.

[4] R. Engelking, General Topology, Sigma Series in Pure Mathematics, vol. 6, Heldermann Verlag, Berlin, 1989.

[5] K. Ishmakhametov, Compactifications of Tychonoff mappings, Ph.D. thesis, Frunze University, Frunze, 1987.

[6] T. Isiwata, Mappings and spaces, Pacific J. Math. 20 (1967), 455-480, correction: ibid. 23 (1967), 630-631.

[7] N. Krolevec, Locally perfect mappings, Dokl. Akad. Nauk SSSR 175 (1967), 1008-1011 (Russian). 
[8] K. D. Magill Jr., The lattice of compactifications of a locally compact space, Proc. London Math. Soc. (3) 18 (1968), 231-244.

[9] G. Nordo, Compattificazioni perfette di funzioni, Ph.D. thesis, Consorzio Università di Messina, Catania, Palermo, 1998.

[10] _ A brief survey on fibrewise general topology, Proceedings of the Meeting on Topological Spaces Theory and Its Applications (Yatsushiro, 1999) (F. Tamari and T. Noiri, eds.), Yatsushiro College of Technology, Yatsushiro, 1999, pp. 39-49.

[11] B. A. Pasynkov, Extension to mappings of certain notions and assertions concerning spaces, Mappings and Functors, Moskov. Gos. Univ., Moscow, 1984, pp. 72-102.

[12] M. C. Rayburn, On Hausdorff compactifications, Pacific J. Math. 44 (1973), no. 2, 707-714.

Giorgio Nordo: Dipartimento di Matematica, Università di Messina, Contrada Papardo, Salita Sperone 31, 98166 Sant'Agata, Messina, Italy

E-mail address: nordo@dipmat.unime.it

Boris A. Pasynkov: Chair of General Topology and Geometry, Faculty of Mechanics and Mathematics, Moscow State University, Moscow 119899, Russia

E-mail address: pasynkovamech.math.msu.su 


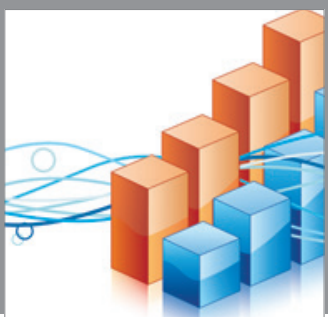

Advances in

Operations Research

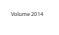

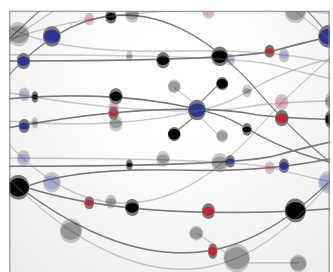

\section{The Scientific} World Journal
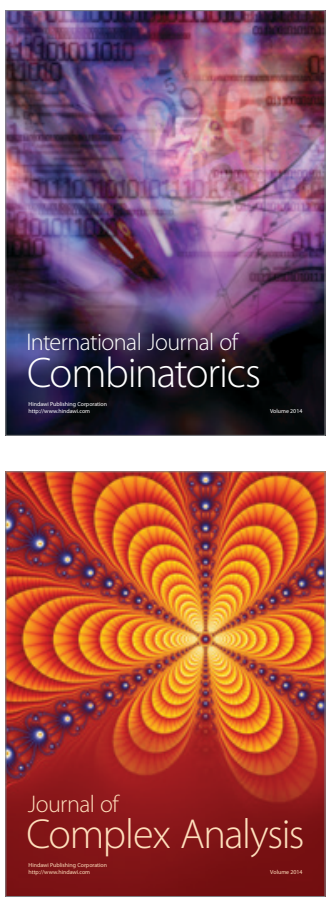

International Journal of

Mathematics and

Mathematical

Sciences
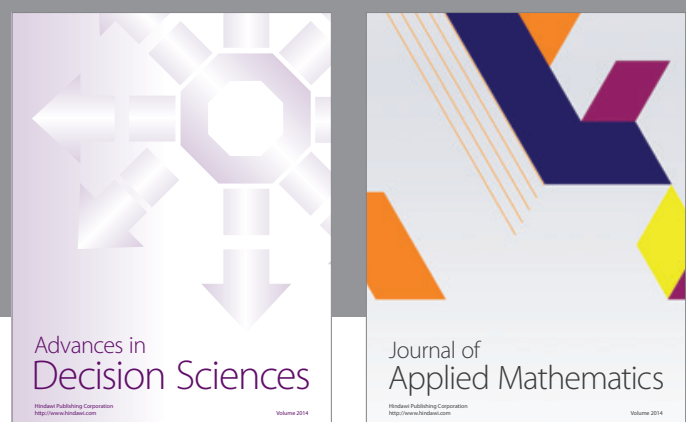

Journal of

Applied Mathematics
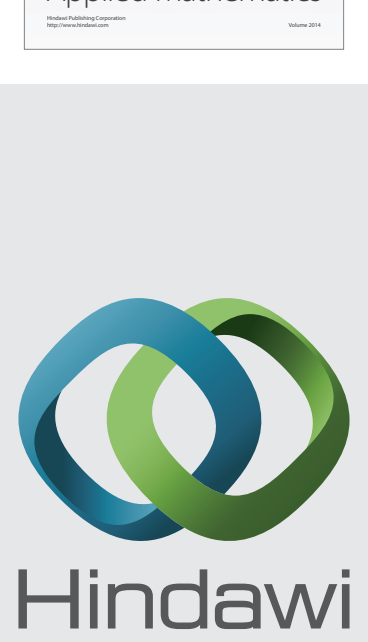

Submit your manuscripts at http://www.hindawi.com
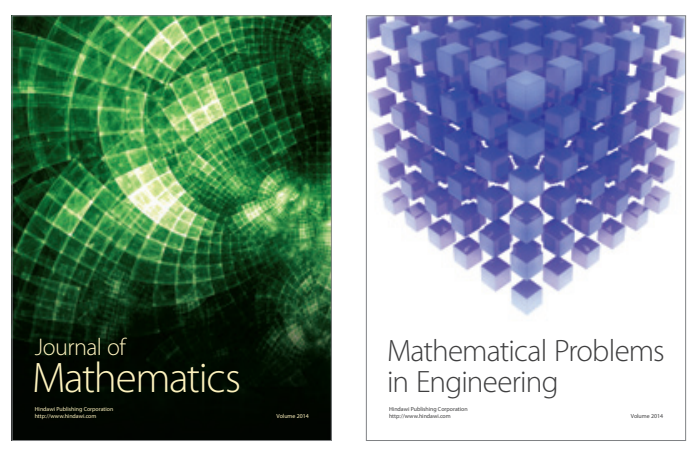

Mathematical Problems in Engineering
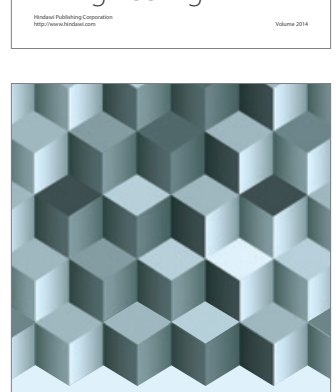

Journal of

Function Spaces
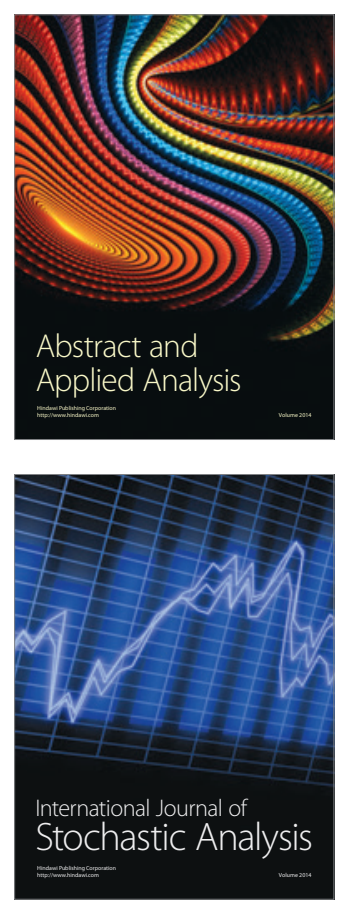

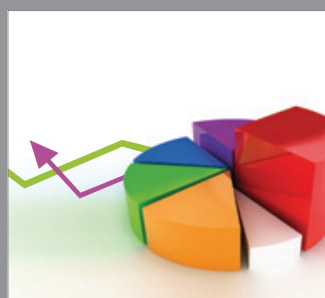

ournal of

Probability and Statistics

Promensencen
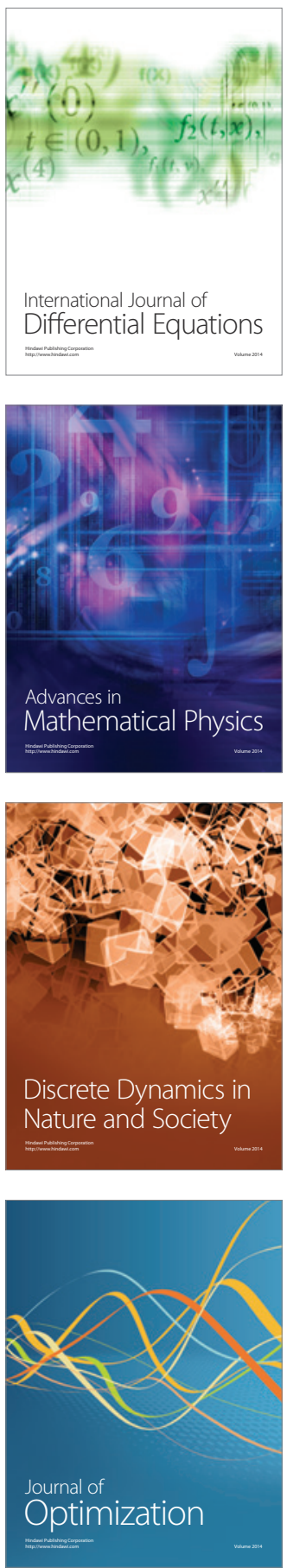